\title{
Quantitative Interpretation of Martensite Microstructure
}

\author{
José Roberto Costa Guimarães ${ }^{\mathrm{a}, \mathrm{c}}$, Paulo Rangel Rios ${ }^{\mathrm{a}, \mathrm{b} *}$ \\ ${ }^{\mathrm{a}}$ Universidade Federal Fluminense, \\ Escola de Engenharia Industrial Metalúrgica de Volta Redonda, \\ Av. dos Trabalhadores, 420, CEP 27255-125, Volta Redonda, RJ, Brasil \\ ${ }^{\mathrm{b}}$ RWTH Aachen University, Institut für Metallkunde und Metallphysik, \\ D-52056 Aachen, Germany \\ ${ }^{\circ}$ Mal. Moura 338H/22C, CEP 06641-000, São Paulo, SP, Brasil
}

Received: November 30, 2010; Revised: January 26, 2011

\begin{abstract}
This work reviews issues and advances a formalism for quantitative interpretation of martensite microstructure, heeding the influence of transformation uniformity and the interaction of martensite with its surroundings. The relationship of volume fraction and number density of martensite units required for kinetics analysis is derived. Additionally we apply the new model to obtain the microstructural path function (MPF) of martensite, and to analyze the autocatalytic spread of the transformation during the martensite burst in Fe-31 wt. (\%) Ni-0.02 wt. (\%) C. The growth of an autocatalytic spread event relates to the chemical driving force, whereas the number of such events relates to the probability of finding a nucleation site to initiate the reaction.
\end{abstract}

Keywords: martensitic phase transformation, kinetics, nucleation, microstructure, analytical methods

\section{Introduction}

The interpretation of microstructure of martensite has both scientific and practical interest because several materials, steels in particular, require management of martensite transformation during processing or utilization to meet advanced engineering requirements/ specifications. Extensively investigated since the $19^{\text {th }}$ century when the name martensite was coined, the microstructure of this transformation is one of its aspects that continues to deserve attention.

Martensite is a displacive transformation accomplished by cooperative atomic displacements, which is disrupted at boundaries between regions of the matrix with different orientations, e.g. a grain boundary. In steels, martensite units propagate under high driving force, grow fast, and maintain their final size without coarsening. These aspects of the transformation signal that the nucleation step controls the kinetics of the reaction. The volume fraction transformed, $V_{V}$, increases with the number density, $N_{V}$, of martensite units. Since the relationship between $V_{V}$ and $N_{V}$ is influenced by the units' arrangement, the crystallography and the morphology of the martensite units, as well as the uniformity of the microstructure are crux. In the present paper we are mainly concerned with the latter aspect, so that the term "unit" is used to stay clear from association with a specific martensite morphology/crystallography.

The classical experiment of Cech and Turnbull ${ }^{1}$ disclosed that the onset of martensite transformation in particulate material is not uniform. Initially it occurs in a few particles, and proceeds by fresh nucleation in still unreacted particles as well as by increasing the fraction transformed in the reacted ones. Thus, it is reasonable to suppose that the particles in which the initial martensite nucleation takes place are "randomly selected" from the untransformed particles². However, in bulk material, the martensite initially formed in a grain sets an autocatalytic process with the grain that can stimulate nucleation across a grain boundary, thus causing local departure from randomness. Figure 1 shows clusters of partially transformed grains in Fe-31 wt. (\%) Ni-0.02 wt. (\%) C cooled immediately below the martensite start temperature ${ }^{3}$.

In a previous work, the present authors obtained an operational $N_{V}=F\left(V_{V}\right)$ relationship that allowed fitting data over a broader range than possible with earlier models by ad hoc applying the extended space concept in spite of the fact that martensite microstructure does not strictly comply with the fundamentals underlying the JMAK's ${ }^{5-7}$ formalism due to departure from uniform randomness of martensite nuclei locations in space. To reconcile results, we review the work described in Guimarães and $\operatorname{Rios}^{4}$ to improve the strength of the advanced model by considering the microstructural uniformity issue in real space. Additionally we demonstrate the use of the microstructural path function (MPF) to analyze the microstructural uniformity and, subsequently, use MPF to characterize the initial autocatalytic spread event, the "martensite burst", in Fe-31 wt. (\%) Ni-0.02 wt. (\%) C.

\section{Experimental Data}

The data used to demonstrate/validate the concepts were described in previous papers of one of the authors as referenced in the text. The material is $\mathrm{Fe}-31$ wt. (\%) Ni-0.02 wt. (\%) $\mathrm{C}$ transforms into lenticular martensite at sub-zero temperatures $(\leq 220 \mathrm{~K})$ so that the transformation microstructure is stable at room temperature and can be expeditiously observed and quantified. For the present purposes, these data were recovered by scanning/digitizing the original publications, and reviewed. To minimize variations from the compilation, the digitized data were consolidated by reiteration and by averaging out small variations on the values of the parameters. Originally, the compiled data did not bear error bars. However the experimental methods, point and intercepts counts on sequential optical photomicrographs (200-500x) were carried systematically to guarantee a statistical error $(95 \%)$ of the mean of $\pm 10 \%$. 


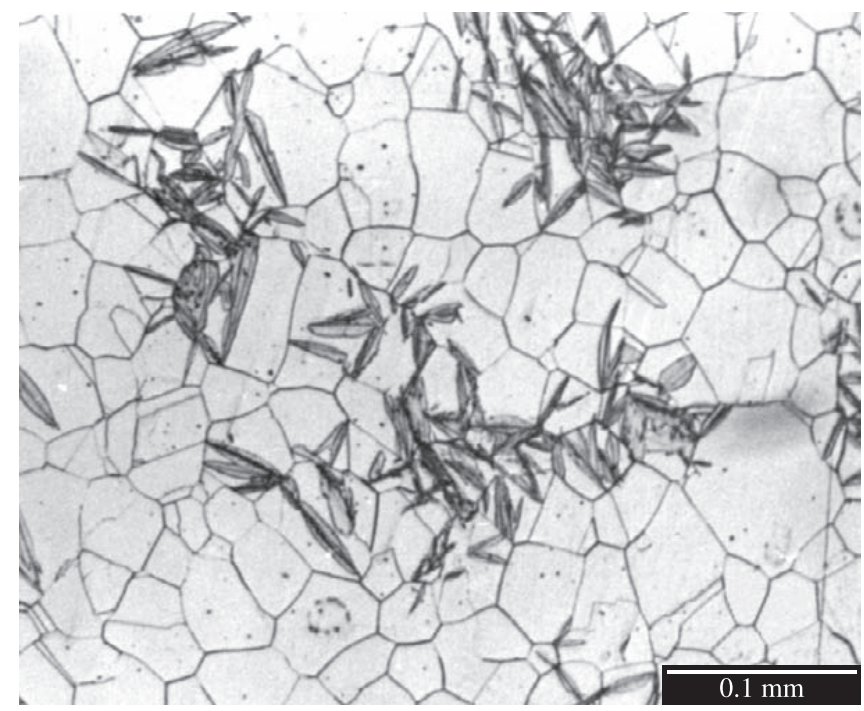

Figure 1. Optical photomicrograph. Fe-31 wt. (\%) Ni-0.02 wt. (\%) C cooled to the burst temperature, $212 \mathrm{~K}$, depicting clusters of partially transformed austenite grains. The austenite grain size (mean intercept length) is equal to $0.026 \mathrm{~mm}$.

\section{The Uniformity Issue}

Independently of how the martensite units are distributed in the material, bearing that the units do not grow or coalesce at the end of the propagation event, the rate of increase of material transformed as martensite nucleation proceeds is proportional to the untransformed volume fraction of austenite,

$$
\frac{d V_{V}}{d N_{V}} \propto\left(1-V_{V}\right)
$$

where $V_{V}$ is volume fraction transformed, and $N_{V}$ the number density of martensite units in the material. To convert Equation 1 into an equality requires consideration of the inhomogeneity of the transformation that includes the interaction of the martensite units with its surroundings. Therefore we introduce an inhomogeneity-adjusted volumetric factor, $\phi\left(N_{V}\right)$, so that formally

$$
d V_{V}=\left(1-V_{V}\right) \phi\left(N_{V}\right) d N_{V}
$$

integration of Equation 2 yields

$$
V_{V}=1-\exp \left(-N_{V} \bar{\phi}\left(N_{V}\right)\right)
$$

where $\bar{\phi}\left(N_{V}\right)$ is defined as

$$
\bar{\phi}\left(N_{V}\right)=\frac{1}{N_{V}} \int_{N_{V}} \phi\left(N_{V}\right) d N_{V}
$$

and it has unit of volume. Thus, for simplicity, we define $\bar{v}_{I A}=\bar{\phi}\left(N_{V}\right)$ and name that the mean inhomogeneity adjusted martensite unit volume, and recast Equation 3

$$
V_{V}=1-\exp \left(-\bar{v}_{I A} N_{V}\right)
$$

it is noticeable that Equation 5 is formally similar to that obtained in Guimarães et al. ${ }^{3}$, however $\bar{v}_{I A}=\bar{\phi}\left(N_{V}\right)$ does not represent the extended volume of martensite. Rather, it is a model parameter whose value is obtainable by inserting experimental values of $V_{V}$ and $N_{V}$ in Equation 5.

At present, we cannot provide an analytical expression for $\bar{v}_{I A}$, however it is apparent that that $\bar{v}_{I A}$ may vary as the transformation proceeds. The simplest phenomenological manner to express that is by way of a linear relationship

$$
\bar{v}_{I A}=v^{*}\left(\left(1-V_{V}\right)+i_{I} V_{V}\right)
$$

where $i_{I}=\frac{v_{0}}{v^{*}} \cdot v_{0}$ and $v^{*}$ are, respectively, the smallest and the largest $\bar{v}_{I A}$. It should be apparent that $v^{*}$ is the volume of the largest martensite unit formed in a grain. $v_{0}$, however, will be determined by the arrangement of the $N_{V}$ martensite units. By combining Equation 6 and Equation 5 we obtain the relationship between $V_{V}$ and $N_{V}$ previously reported ${ }^{4}$ but free from assuming that martensite complies with the fundamental underlying the JMAK' $\mathrm{s}^{5-7}$ formalism.

$$
N_{V}=\frac{\ln \left(1-V_{V}\right)^{-1}}{v^{*}\left(1-V_{V}\right)+i_{I} \cdot V_{V}}
$$
The mean volume of the martensite units $\bar{v}=\frac{V_{V}}{N_{V}}$ can be obtained
by using Equation 7 in Equation 5,

$$
\bar{v}=v * \frac{V_{V}\left(\left(1-V_{V}\right)+i_{I} V_{V}\right)}{\ln \left(1-V_{V}\right)^{-1}}
$$

To check the usefulness of the model we refer to Figure 2 that depicts values of $\bar{v}$ and $V_{V}$ typical of Fe-31 wt. (\%) Ni-0.02 wt. (\%) $\mathrm{C}$ possessing grain sizes(mean intercept length) ranging from 0.026 to $0.142 \mathrm{~mm}$, and transformed by cooling. The lines through the data were obtained with Equation 8 by fitting and $v^{*}$. The following values of $v^{*}=1.0 \cdot 10^{-4}, 1.7 \cdot 10^{-5}$ and $1.0 \cdot 10^{-5} \mathrm{~mm}^{3}$ were used to visually describe the data typical of the material with mean austenite grain intercepts of $0.142,0.049$ and $0.026 \mathrm{~mm}$, respectively.

Inspection of Figure 2 shows that $i_{I}=0$ suits the steeper variation of the mean martensite unit volume in the coarser grained condition, whereas $i_{I}=1$ describes better the more discreet variation of $\bar{v}$ in materials with medium and fine grain sizes. Noteworthy, the coarse grained material transformation is well spread over the austenite grains, whereas fine and the medium grained material exhibit untransformed austenite grains up to $V_{V}=0.4-0.5$. For the reasons discussed in $\mathrm{Magee}^{8}$, until the reaction is all over the austenite grains the mean plate volume remains nearly constant. Therefore, it is apparent that the parameter $i_{I}$ provides indication of the lack of uniformity of the microstructure.

\section{The Microstructural Path Function of Martensite}

The MPF is an important tool to analyze property-microstructure correlation as well as fundamental aspects of phase transformations ${ }^{9}$, such as the rate of autocatalytic martensite nucleation ${ }^{10-15}$. The microstructural path function (MPF) expresses the volume density of the product-matrix interfaces as a function of the fraction transformed, $S_{V}^{M-\gamma}=f\left(V_{V}\right)$. In a martensite-austenite material, recalling that martensite units do not coalesce or coarsen, the total surface area of the martensite units is proportional to the number of units per unit volume as

$$
S_{V}^{M}=\bar{s} N_{V}
$$

where $\bar{s}$ is the mean surface area of the martensite units. Thus, subtracting the area associated with martensite-martensite contacts gives us the area of free martensite-austenite interfaces,

$$
S_{v}^{M-\gamma}=N_{V} \bar{S}\left(1-V_{V}\right)
$$

Calling back Equation 5 and Equation 7, and taking $\bar{s} \propto(\bar{v})^{2 / 3}$ one obtains 


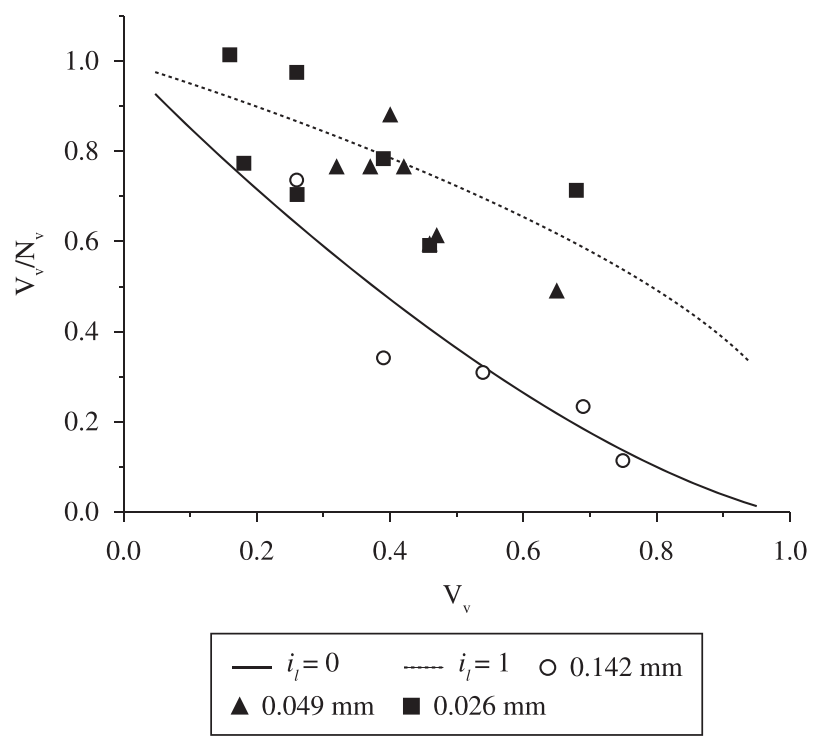

Figure 2. Martensite mean plate volume in Fe-31 wt. (\%) Ni-0.02 wt. (\%) C normalized with respect to $v^{*}$ plotted against the fraction transformed. The grain size (mean intercept length) is displayed in the graph.

$$
S_{V}^{M-\gamma}=\Gamma\left(1-V_{V}\right) \ln \frac{1}{1-V_{V}}
$$

where $\Gamma=\Gamma_{0} \cdot(\bar{v})^{-1 / 3}$, and $\Gamma_{0}$ is a unitless constant. Using Equation 8 in Equation 11 yields the MPF

$$
S_{V}^{M-\gamma}=\Gamma_{0} \frac{\left(1-V_{V}\right)\left(\ln \frac{1}{1-V_{V}}\right)^{4 / 3}}{\left(V_{V}\right)^{1 / 3}\left(\left(1-V_{V}\right)+i_{I} V_{V}\right)^{1 / 3}}
$$

Note that Equation 12 like Equation 8 has two fitting parameters, one of which, $i_{I}$, is common to both equations. Application of the MPF concept to lenticular martensite in Fe-31 wt. (\%) Ni-0.02 wt. (\%) C is demonstrated in Figure 3. It is noted that the data characteristic of material transformed by cooling below the burst temperature, $M_{B}{ }^{16}$, or mechanical induced $23 \mathrm{~K}$ above the $M_{B}{ }^{17}$ are well described by Equation 12, using $i_{I}=0$ for the coarse grained $(0.142 \mathrm{~mm})$ condition, and $i_{I}=1$ for the medium-fine $(0.059-0.021 \mathrm{~mm})$ materials. In the former, the martensite is well spread, whereas in the latter the martensite units are found inside clusters of partially transformed grains. The following values of $\Gamma_{0}$ were used to fit the data with the correlation coefficients indicated: $91.5 \mathrm{~mm}^{-1}$ and $R^{2}=0.93$ $(0.142 \mathrm{~mm}), 113.1 \mathrm{~mm}^{-1}$ and $R^{2}=0.50(0.059 \mathrm{~mm})$ and $207.4 \mathrm{~mm}^{-1}$ and $R^{2}=0.90(0.021 \mathrm{~mm})$. The fitting for medium grain size material has a low correlation coefficient for $i_{I}=1$. This is to be expected because if for large grain sizes: $i_{I}=0$, and for fine grain sizes: $i_{I}=1$, one might expect $i_{I}$ to be between these values and thus lower correlation to be obtained by assuming $i_{I}=1$. Unfortunately one would need more data on intermediate grain to be able to determine $i_{I}$ more precisely but for lack of data $i_{I}=1$ was used.

\section{The Microstructural Path Function of the Spread of Martensite}

As previously described, the initial martensite nucleation events occur in randomly scattered grains ${ }^{1,11}$. Autocatalysis promotes additional transformation within these grains and fosters the

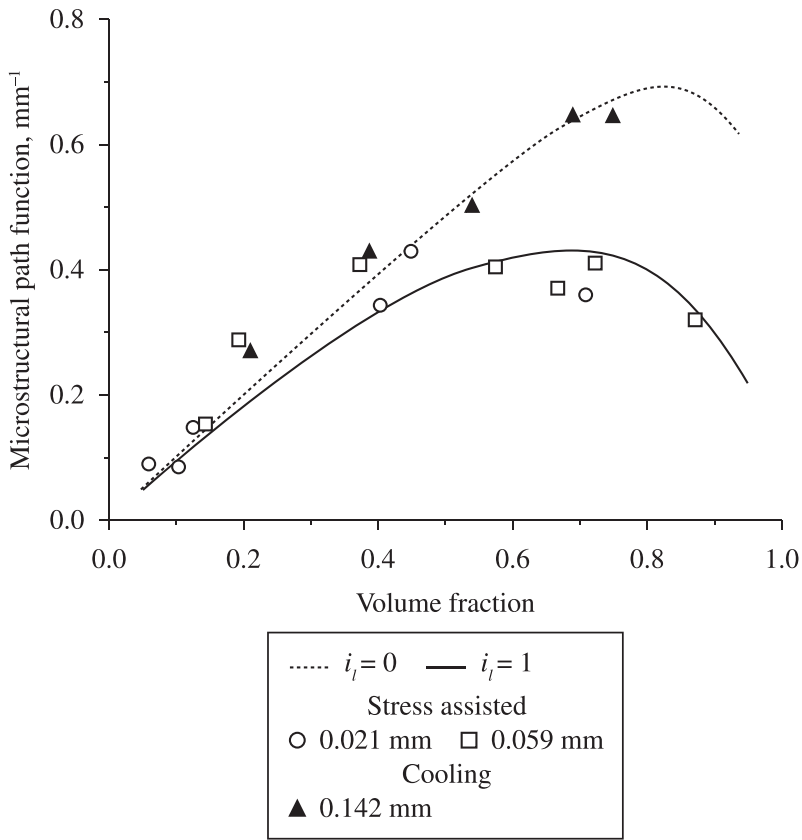

Figure 3. Microstructural path function of Fe-31 wt. (\%) Ni-0.02 wt. (\%) C transformed by cooling ${ }^{16}$ or stress assisted ${ }^{17}$ (elastic straining) above the $M_{S}$ temperature plotted after Equation 12.

impingement of martensite units on grain boundaries, causing nucleation in a next grain and so forth, forming a cluster of partially transformed grains. The collection of such local spread events formed at the martensite start temperature comprises the burst which signals the beginning of martensite transformation in some alloys, e.g. Fe-31 wt. (\%) Ni-0.02 wt. (\%) C.

The clusters of grains produced by the spread events are delimited by ordinary austenite grain boundaries. These clusters do not grow by the motion of grain boundaries. Instead, fresh martensite nucleation in untransformed grains causes new clusters to form. These spread events adhere by impingement yielding a larger cluster. Since a spread event starts at random, reflecting the initial nucleation of martensite, the rate of increase of the volume density of grains partially transformed, $N_{V G}$, with increasing the number density of martensite units located within spread events, $N_{V, S P}$, is limited by the volume fraction of material in the untransformed grains,

$$
\frac{d N_{V G}}{d N_{V, S P}}=\left(1-V_{V G}\right)
$$

where $V_{V G}$ is the volume fraction of material in the reacted grains (grains partially transformed to martensite) that comprise the overall spread.

However, not every martensite unit in a spread event is capable of inducting the reaction into a next grain since it can be barred by other units from impinging on a grain boundary, or by the arrest of the midrib propagation as seen in isothermal martensite transformation ${ }^{17,19}$. Additionally, the efficacy of the impingement on grain boundary to poke nucleation in the next grain is counterbalanced by the elastic interaction of the martensite units within the transforming grains that can promotes self-accommodation ${ }^{20}$ and decreases the energy of the system. Therefore, to account for these competing factors, we introduce the probability that a martensite unit pokes nucleation across a grain boundary, $P$, writing

$$
d N_{V, S P}=P d N_{V}
$$


Using Equation 14 in Equation 13, and recalling that $V_{V G}=q N_{V, S P}$, where $q$ is the mean austenite grain volume, followed by integration give us an expression for $V_{V G}$

$$
V_{V G}=1-\exp \left(-q P N_{V}\right)
$$

This equation is analogous to the empirical equation earlier used describe the spread of martensite in Fe-31 wt. (\%) Ni-0.02 wt. (\%) $\mathrm{C}$ with a high correlation, by fitting $P=0.09^{21}$.

Noteworthy, the analysis of the spread of isothermally transformed FeNiMn alloy ${ }^{16}$ indicates that $P$ plummets if the austenite grain size is much larger than the maximum attainable martensite unit diameter ${ }^{19}$, because the impingement of martensite on grain boundaries may become less frequent.

To proceed with the analysis we recall that a spread event starts at random so that the microstructural path concept can be applied to estimate the maximum attainable cluster size formed in a burst, $\lambda_{C, X}^{B}$, by ignoring impingement,

$$
\lambda_{C, X}^{B}=\frac{4 V_{V G, X}^{B}}{S_{V G, X}^{B}}
$$

where $V_{V G, X}^{B}$ and $S_{V G, X}^{B}$ are extended quantities ${ }^{5-7}$, respectively, the volume fraction and the volume density of cluster surface area per unit volume of material. It should be apparent that the impingement compensate cluster size, $\lambda_{C, X}^{B}$, relates to the energy content of the autocatalysis ${ }^{21}$. From a microstructural point of view, $\lambda_{C, X}^{B}$ bears analogy with the mean impingement compensated grain size discussed in ${ }^{23}$.

Since the spread events that comprise the burst start at uniform randomly located sites, the relationship between the extended volume and the volume fraction of clusters may be written as ${ }^{5-7,24}$

$$
V_{V G}^{B}=1-\exp \left(-V_{V G, X}^{B}\right)
$$

and

$$
S_{V G}^{B}=S_{V G, X}^{B}\left(1-V_{V G}^{B}\right)
$$

Combining Equation 17 and Equation 18, one obtains the microstructural path function for the spread of the martensite burst that allows obtaining $\lambda_{C, X}^{B}$ from measurable metallographic parameters.

$$
\lambda_{C, X}^{B}=\frac{4\left(1-V_{V G}^{B}\right) \cdot \ln \left(1-V_{V G}^{B}\right)^{-1}}{S_{V G}^{B}}
$$

Table 1 shows the calculated values of $\lambda_{C, X}^{B}$ and the burst temperature, $\mathrm{M}_{\mathrm{B}}$, observed with Fe-31 wt. (\%) Ni-0.02 wt. (\%) $\mathrm{C}$ with austenitic grain sizes, $\lambda{ }^{\gamma}$ (mean intercept length), ranging from 0.026 to $0.142 \mathrm{~mm}$. Included in Table 1 for comparison are the value of the real cluster size, $\lambda_{C}^{B}$, determined from the microstructure.

Inspection of Table 1 indicates that the variation of both $\lambda_{C, X}^{B}$ $(0.25-0.30 \mathrm{~mm})$ and $\mathrm{M}_{\mathrm{B}}(212-222 \mathrm{~K})$ is rather meek compared with

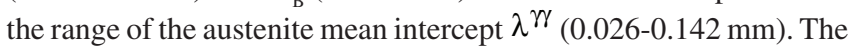
actual cluster size, $\lambda_{C}^{B}$, in fine to medium grained material are in line with the values of $\lambda_{C, X}^{B}$. However, in the coarser grained condition, $\lambda_{C}^{B} \gg \lambda_{C, X}^{B}$ evidencing the geometric effect of cluster adherence on impingement.Henceforth the "growth" of a spread event, estimated by $\lambda_{C, X}^{B}$ depends on the available driving force, whereas the "nucleation" relates to the number density of preferred nucleation sites to start the transformation. Thus, $\lambda_{C, X}^{B}$ may be considered an estimator of the energy content of a spread event. Merely as reference, the
Table 1. Fe-31 wt. (\%) Ni-0.02 wt. (\%) C burst data ${ }^{14}$.

\begin{tabular}{cccc}
\hline$\lambda \gamma(\mathrm{mm})$ & $\mathrm{M}_{\mathrm{B}}(\mathrm{K})$ & $\lambda_{C, X}^{B}(\mathrm{~mm})$ & $\lambda_{C}^{B}(\mathrm{~mm})$ \\
\hline 0.026 & 212 & 0.26 & 0.28 \\
0.029 & 214 & 0.29 & 0.31 \\
0.049 & 220 & 0.28 & 0.33 \\
0.064 & 222 & 0.25 & 0.44 \\
0.143 & 220 & 0.30 & 0.86 \\
\hline
\end{tabular}

estimated contribution of autocatalysis to the spread of martensite in a polycrystalline $\mathrm{Fe}-24.4$ wt. (\%) Ni- 0.44 wt. (\%) C is about $10 \%$ of the chemical driving force at the burst temperature ${ }^{22}$. Last, it should be clear that $\lambda_{C, X}^{B}$ reflects the probability, $P$, that a martensite unit pokes nucleation across a grain boundary contained in $V_{V G}^{B}$, Equation 15 .

\section{Summary and Conclusions}

We have described a formalism to relate the volume fraction of martensite to the number of martensite units, required to analyze the kinetic aspects of the transformation, that takes into consideration the lack of uniform randomness of the reaction that typifies athermal, isothermal and mechanical induced martensite transformations $\mathrm{s}^{1,3,17,18,24,25}$. A parameter, $i_{I}$ was introduced that reflects the uniformity of the microstructure.

The usefulness of the development was demonstrated with transformation data obtained with Fe-31 wt. (\%) Ni-0.02 wt. (\%) $\mathrm{C}$ for convenience. However the formalism does not depend on the morphology/crystallographic aspects of the martensite units, and can be readily applied to obtain the microstructural path function (MPF) for the martensite transformation as demonstrated.

Additionally we reviewed the autocatalytic spread of martensite, and applied the concept of compensated cluster's mean intercept, $\lambda_{C, X}^{B}$, defined by Equation 19 to estimate the extent of material reached by the autocatalytic spread initiated in an austenite grain. The results, typical of Fe-31 wt. (\%) Ni-0.02 wt. (\%) C, suggest that as might be expected the potential of the spread event that yield a clusters of partially reacted grains derives mainly from the chemical driving force. The behavior of Fe-31 wt. (\%) Ni-0.02 wt. (\%) C, supports the contention that, the growth of a spread event relates mainly from the chemical driving force, whereas the number of such events depends on the probability of finding a nucleation site to initiate the reaction. The latter are found more scattered in fine grained austenite ${ }^{10}$.

\section{Acknowledgements}

P. R. Rios is grateful to Conselho Nacional de Desenvolvimento Científico e Tecnológico, CNPq, and to Fundação de Amparo à Pesquisa do Estado do Rio de Janeiro, FAPERJ, for financial support. P. R. Rios would also like to thank the Humboldt Foundation for the Humboldt Research Award and Professor Günter Gottstein for his hospitality during the author's stay at Institut für Metallkunde and Metallphysik of RWTH-Aachen where this work has been done.

\section{References}

1. Cech RE and Turnbull D. Heterogeneous nucleation of the martensite transformation. Transactions AIME. 1956; 206(2):124-132.

2. Cohen M and Olson GB. Martensitic nucleation and the role of the nucleation defect. Supplement Transactions Japan Institute of Metals. 1976; 17:93-98 
3. Guimarães JRC and Gomes JC. Metallographic study of influence of austenite grain-size on martensite kinetics in Fe-31.9 Ni-0.02C. Acta Metallurgica. 1978; 26(10):1591-1596.

4. Guimarães JRC and Rios PR. Unified Model for Plate and Lath Martensite with Athermal Kinetics. Metallurgical and Materials Transactions A. 2010; 41(8):1928-1935

5. Johnson WA and Mehl RF. Reaction kinetics in processes of nucleation and growth. Transactions AIME. 1939; 135:416-441.

6. Avrami MJ. Kinetics of phase change I general theory. The Journal of Chemical Physics. 1939; 7(12):1103-1112.

7. Kolmogorov NA. The statistics of crystal growth in metals. Isvestiia Academii Nauk SSSR - Seriia Matematicheskaia. 1937; 1:333-359.

8. Magee CL. The nucleation of martensite. In: Aaronson HI, editor. Phase transformations. Ohio: ASM International, Metals Park; 1968.

9. Vandermeer RA, Masumura RA and Rath B. Microstructural paths of shape-preserved nucleation and growth transformations. Acta Metallurgica et Materialia. 1991; 39(3):383-389.

10. Guimarães JRC and Rios PR. Driving force and thermal activation in martensite kinetics. Metallurgical and Materials Transactions A. 2009; 40(10):2255-2509.

11. Guimarães JRC and Rios PR. Martensite start temperature and the austenite grain-size. Journal Materials Science. 2010; 45(4):1074-1077.

12. Guimarães JRC and Rios PR. Initial nucleation kinetics of martensite transformation. Journal Materials Science. 2008; 43:5206-5210.

13. Rios PR and Guimarães JRC. Microstructural path analysis of athermal martensite. Scripta Materialia. 2007; 57(12):1105-1108.

14. Rios PR and Guimarães JRC. Formal analysis of isothermal martensite spread. Materials Research. 2008; 11(1):103-108.
15. Rios PR and Guimarães JRC. Microstructural path analysis of martensite burst. Materials Research. 2010; 13(1):119-124.

16. Guimarães JRC and Gomes JC. Microstructural aspects of martensite transformation in coarse-grained Fe-31.1 pct Ni-0.02 pct C. Metallurgical and Materials Transactions A. 1979; 10(1):109-112.

17. Leal RH and Guimarães JRC. Microstructure evolution during mechanically induced martensitic-transformation in Fe-31-percent Ni-0.1-percent-C. Materials Science and Engineering. 1981; 48(2):249-254.

18. Ghosh G. Spread of transformation and plate dimensions of isothermally formed martensite. Materials Science and Engineering. 1988; 101:213-220.

19. Guimarães JRC. Isothermal martensite: austenite grain size and kinetics of 'spread'. Materials Science and Technology 2008, 24(7):843-847.

20. Bokros JC and Parker ER. Mechanism of martensite burst transformation in Fe-Ni single crystals. Acta Metallurgica. 1963; 11(12):1291-1301.

21. Guimarães JRC and Saavedra A. A computer-assisted analysis of the spread of martensite-transformation. Materials Science and Engineering 1984; 62(1):11-15.

22. Knorovsky GA. Autocatalysis of martensite transformation [D.Sc. thesis]. Massachusetts Institute of Technology; 1977.

23. Vandermeer RA. Microstructural descriptors and the effects of nuclei clustering on recrystallization path kinetics. Acta Materialia. 2005 53(5):1449-1457.

24. Rios PR and Villa E. Transformation kinetics for inhomogeneous nucleation. Acta Materialia. 2009; 57(4):1199-1208.

25. Raghavan V. Formation sequence of plates in isothermal martensite transformation. Acta Metallurgica. 1969; 17(10):1299-1303. 